\title{
Products and sums divisible by central binomial coefficients
}

\author{
Zhi-Wei Sun* \\ Department of Mathematics \\ Nanjing University \\ Nanjing 210093, People's Republic of China \\ zwsun@nju.edu.cn
}

Submitted: May 20, 2011; Accepted: Jan 5, 2013; Published: Jan 18, 2013

Mathematics Subject Classifications: 05A10, 11B65, $11 \mathrm{~A} 07$.

\begin{abstract}
In this paper we study products and sums divisible by central binomial coefficients. We show that

$$
2(2 n+1)\left(\begin{array}{c}
2 n \\
n
\end{array}\right) \mid\left(\begin{array}{c}
6 n \\
3 n
\end{array}\right)\left(\begin{array}{c}
3 n \\
n
\end{array}\right) \text { for all } n=1,2,3, \ldots
$$
\end{abstract}

Also, for any nonnegative integers $k$ and $n$ we have

$$
\left(\begin{array}{c}
2 k \\
k
\end{array}\right) \mid\left(\begin{array}{c}
4 n+2 k+2 \\
2 n+k+1
\end{array}\right)\left(\begin{array}{c}
2 n+k+1 \\
2 k
\end{array}\right)\left(\begin{array}{c}
2 n-k+1 \\
n
\end{array}\right)
$$

and

$$
\left(\begin{array}{c}
2 k \\
k
\end{array}\right) \mid(2 n+1)\left(\begin{array}{c}
2 n \\
n
\end{array}\right) C_{n+k}\left(\begin{array}{c}
n+k+1 \\
2 k
\end{array}\right),
$$

where $C_{m}$ denotes the Catalan number $\frac{1}{m+1}\left(\begin{array}{c}2 m \\ m\end{array}\right)=\left(\begin{array}{c}2 m \\ m\end{array}\right)-\left(\begin{array}{c}2 m \\ m+1\end{array}\right)$. On the basis of these results, we obtain certain sums divisible by central binomial coefficients.

Keywords: central binomial coefficients; divisibility; congruences

\section{Introduction}

Central binomial coefficients are given by $\left(\begin{array}{c}2 n \\ n\end{array}\right)$ with $n \in \mathbb{N}=\{0,1,2, \ldots\}$. The Catalan numbers

$$
C_{n}=\frac{1}{n+1}\left(\begin{array}{c}
2 n \\
n
\end{array}\right)=\left(\begin{array}{c}
2 n \\
n
\end{array}\right)-\left(\begin{array}{c}
2 n \\
n+1
\end{array}\right)(n=0,1,2, \ldots)
$$

\footnotetext{
${ }^{*}$ Supported by the National Natural Science Foundation (grant 11171140) of China and the PAPD of Jiangsu Higher Education Institutions.
} 
play important roles in combinatorics (cf. R. P. Stanley [13, pp. 219-229]). There are many sophisticated congruences involving central binomial coefficients and Catalan numbers (see, e.g., $[15,18,19])$.

In 1998 N. J. Calkin [5] proved that $\left(\begin{array}{c}2 n \\ n\end{array}\right) \mid \sum_{k=-n}^{n}(-1)^{k}\left(\begin{array}{c}2 n \\ n+k\end{array}\right)^{m}$ for any $m, n \in \mathbb{Z}^{+}$. See also V.J.W. Guo, F. Jouhet and J. Zeng [9], and H. Q. Cao and H. Pan [6] for further extensions of Calkin's result.

In this paper we investigate a new kind of divisibility problems involving central binomial coefficients.

Our first theorem is as follows.

Theorem 1. (i) For any positive integer $n$ we have

$$
2(2 n+1)\left(\begin{array}{c}
2 n \\
n
\end{array}\right) \mid\left(\begin{array}{c}
6 n \\
3 n
\end{array}\right)\left(\begin{array}{c}
3 n \\
n
\end{array}\right) .
$$

(ii) Let $k$ and $n$ be nonnegative integers. Then

$$
\left(\begin{array}{c}
2 k \\
k
\end{array}\right) \mid\left(\begin{array}{c}
4 n+2 k+2 \\
2 n+k+1
\end{array}\right)\left(\begin{array}{c}
2 n+k+1 \\
2 k
\end{array}\right)\left(\begin{array}{c}
2 n-k+1 \\
n
\end{array}\right)
$$

and

$$
\left(\begin{array}{c}
2 k \\
k
\end{array}\right) \mid(2 n+1)\left(\begin{array}{c}
2 n \\
n
\end{array}\right) C_{n+k}\left(\begin{array}{c}
n+k+1 \\
2 k
\end{array}\right) .
$$

In view of (1) it is worth introducing the sequence

$$
S_{n}=\frac{\left(\begin{array}{c}
6 n \\
3 n
\end{array}\right)\left(\begin{array}{c}
3 n \\
n
\end{array}\right)}{2(2 n+1)\left(\begin{array}{c}
2 n \\
n
\end{array}\right)} \quad(n=1,2,3, \ldots) .
$$

Here we list the values of $S_{1}, \ldots, S_{8}$ :

5, 231, 14568, 1062347, 84021990,

$$
\text { 7012604550, 607892634420, 54200780036595. }
$$

The author generated this sequence as A176898 at N.J.A Sloane's OEIS (cf. [16]). By Stirling's formula, $S_{n} \sim 108^{n} /(8 n \sqrt{n \pi})$ as $n \rightarrow+\infty$. Set $S_{0}=1 / 2$. Using Mathematica we find that

$$
\sum_{k=0}^{\infty} S_{k} x^{k}=\frac{\sin \left(\frac{2}{3} \arcsin (6 \sqrt{3 x})\right)}{8 \sqrt{3 x}} \quad\left(0<x<\frac{1}{108}\right)
$$

and in particular

$$
\sum_{k=0}^{\infty} \frac{S_{k}}{108^{k}}=\frac{3 \sqrt{3}}{8}
$$

Mathematica also yields that

$$
\sum_{k=0}^{\infty} \frac{S_{k}}{(2 k+3) 108^{k}}=\frac{27 \sqrt{3}}{256} .
$$


It would be interesting to find a combinatorial interpretation or recursion for the sequence $\left\{S_{n}\right\}_{n \geqslant 1}$.

One can easily show that $S_{p} \equiv 15-30 p+60 p^{2}\left(\bmod p^{3}\right)$ for any odd prime $p$. Below we present a conjecture concerning congruence properties of the sequence $\left\{S_{n}\right\}_{n \geqslant 1}$.

Conjecture 2. (i) Let $n \in \mathbb{Z}^{+}=\{1,2,3, \ldots\}$. Then $S_{n}$ is odd if and only if $n$ is a power of two. Also, $3 S_{n} \equiv 0(\bmod 2 n+3)$.

(ii) For any prime $p>3$ we have

$$
\sum_{k=1}^{p-1} \frac{S_{k}}{108^{k}} \equiv \begin{cases}0(\bmod p) & \text { if } p \equiv \pm 1(\bmod 12), \\ -1(\bmod p) & \text { if } p \equiv \pm 5(\bmod 12) .\end{cases}
$$

Remark 3. Part (i) of Conjecture 2 might be shown by our method for proving Theorem $1(\mathrm{i})$.

Our following conjecture is concerned with a companion sequence of $\left\{S_{n}\right\}_{n \geqslant 0}$.

Conjecture 4 . There are positive integers $T_{1}, T_{2}, T_{3}, \ldots$ such that

$$
\sum_{k=0}^{\infty} S_{k} x^{2 k+1}+\frac{1}{24}-\sum_{k=1}^{\infty} T_{k} x^{2 k}=\frac{\cos \left(\frac{2}{3} \arccos (6 \sqrt{3} x)\right)}{12}
$$

for all real $x$ with $|x| \leqslant 1 /(6 \sqrt{3})$. Also, $T_{p} \equiv-2(\bmod p)$ for any prime $p$.

Here we list the values of $T_{1}, \ldots, T_{8}$ :

$$
\begin{gathered}
1,32,1792,122880,9371648, \\
763363328,65028489216,5722507051008 .
\end{gathered}
$$

In 1914 Ramanujan [12] obtained that

$$
\sum_{k=0}^{\infty} \frac{4 k+1}{(-64)^{k}}\left(\begin{array}{c}
2 k \\
k
\end{array}\right)^{3}=\frac{2}{\pi}
$$

and

$$
\sum_{k=0}^{\infty}(20 k+3) \frac{\left(\begin{array}{c}
2 k \\
k
\end{array}\right)^{2}\left(\begin{array}{c}
4 k \\
2 k
\end{array}\right)}{\left(-2^{10}\right)^{k}}=\frac{8}{\pi}
$$

(See also $[2,3,4]$ for such series.) Actually the first identity was originally proved by G. Bauer in 1859. Both identities can be proved via the WZ (Wilf-Zeilberger) method (cf. M. Petkovšek, H. S. Wilf and D. Zeilberger [11], and Zeilberger [21] for this method). For WZ proofs of the two identities, see S. B. Ekhad and D. Zeilberger [7] and Guillera [8]. van Hamme [20] conjectured that the first identity has a $p$-adic analogue. This conjecture was first proved by E. Mortenson [10], and recently re-proved in [22] via the WZ method.

On the basis of Theorem 1, we deduce the following new result. 
Theorem 5. For any positive integer $n$ we have

$$
4(2 n+1)\left(\begin{array}{c}
2 n \\
n
\end{array}\right) \mid \sum_{k=0}^{n}(4 k+1)\left(\begin{array}{c}
2 k \\
k
\end{array}\right)^{3}(-64)^{n-k}
$$

and

$$
4(2 n+1)\left(\begin{array}{c}
2 n \\
n
\end{array}\right) \mid \sum_{k=0}^{n}(20 k+3)\left(\begin{array}{c}
2 k \\
k
\end{array}\right)^{2}\left(\begin{array}{c}
4 k \\
2 k
\end{array}\right)\left(-2^{10}\right)^{n-k}
$$

Now we pose two more conjectures.

Conjecture 6. (i) For any $n \in \mathbb{Z}^{+}$we have

$$
a_{n}:=\frac{1}{8 n^{2}\left(\begin{array}{c}
2 n \\
n
\end{array}\right)^{2}} \sum_{k=0}^{n-1}\left(205 k^{2}+160 k+32\right)(-1)^{n-1-k}\left(\begin{array}{c}
2 k \\
k
\end{array}\right)^{5} \in \mathbb{Z}^{+} .
$$

(ii) Let $p$ be an odd prime. If $p \neq 3$ then

$$
\sum_{k=0}^{(p-1) / 2}\left(205 k^{2}+160 k+32\right)(-1)^{k}\left(\begin{array}{c}
2 k \\
k
\end{array}\right)^{5} \equiv 32 p^{2}+\frac{896}{3} p^{5} B_{p-3}\left(\bmod p^{6}\right)
$$

where $B_{0}, B_{1}, B_{2}, \ldots$ are Bernoulli numbers. If $p \neq 5$ then

$$
\sum_{k=0}^{p-1}\left(205 k^{2}+160 k+32\right)(-1)^{k}\left(\begin{array}{c}
2 k \\
k
\end{array}\right)^{5} \equiv 32 p^{2}+64 p^{3} H_{p-1}\left(\bmod p^{7}\right)
$$

where $H_{p-1}=\sum_{k=1}^{p-1} 1 / k$.

Remark 7. . Note that $a_{1}=1$ and

$$
4(2 n+1)^{2} a_{n+1}+n^{2} a_{n}=\left(205 n^{2}+160 n+32\right)\left(\begin{array}{c}
2 n-1 \\
n
\end{array}\right)^{3} \text { for } n=1,2, \ldots
$$

The author generated the sequence $\left\{a_{n}\right\}_{n>0}$ at OEIS as A176285 (cf. [16]). In $1997 \mathrm{~T}$. Amdeberhan and D. Zeilberger [1] used the WZ method to obtain

$$
\sum_{k=1}^{\infty} \frac{(-1)^{k}\left(205 k^{2}-160 k+32\right)}{k^{5}\left(\begin{array}{c}
2 k \\
k
\end{array}\right)^{5}}=-2 \zeta(3) .
$$

Conjecture 8. (i) For any odd prime $p$, we have

$$
\sum_{k=0}^{p-1} \frac{28 k^{2}+18 k+3}{(-64)^{k}}\left(\begin{array}{c}
2 k \\
k
\end{array}\right)^{4}\left(\begin{array}{c}
3 k \\
k
\end{array}\right) \equiv 3 p^{2}-\frac{7}{2} p^{5} B_{p-3}\left(\bmod p^{6}\right)
$$


and

$$
\sum_{k=0}^{(p-1) / 2} \frac{28 k^{2}+18 k+3}{(-64)^{k}}\left(\begin{array}{c}
2 k \\
k
\end{array}\right)^{4}\left(\begin{array}{c}
3 k \\
k
\end{array}\right) \equiv 3 p^{2}+6\left(\frac{-1}{p}\right) p^{4} E_{p-3}\left(\bmod p^{5}\right),
$$

where $E_{0}, E_{1}, E_{2}, \ldots$ are Euler numbers.

(ii) For any integer $n>1$, we have

$$
\sum_{k=0}^{n-1}\left(28 k^{2}+18 k+3\right)\left(\begin{array}{c}
2 k \\
k
\end{array}\right)^{4}\left(\begin{array}{c}
3 k \\
k
\end{array}\right)(-64)^{n-1-k} \equiv 0\left(\bmod (2 n+1) n^{2}\left(\begin{array}{c}
2 n \\
n
\end{array}\right)^{2}\right) .
$$

Also,

$$
\sum_{k=1}^{\infty} \frac{\left(28 k^{2}-18 k+3\right)(-64)^{k}}{k^{5}\left(\begin{array}{c}
2 k \\
k
\end{array}\right)^{4}\left(\begin{array}{c}
3 k \\
k
\end{array}\right)}=-14 \zeta(3) .
$$

Remark 9. The conjectural series for $\zeta(3)=\sum_{n=1}^{\infty} 1 / n^{3}$ was first announced by the author in a message to Number Theory Mailing List (cf. [17]) on April 4, 2010.

For more conjectures similar to Conjectures 6 and 8 the reader may consult [14] and [16].

In the next section we will establish three auxiliary inequalities involving the floor function. Sections 3 and 4 are devoted to the proofs of Theorem 1 and Theorem 5 respectively.

\section{Three auxiliary inequalities}

In this section, for a rational number $x$ we let $\{x\}=x-\lfloor x\rfloor$ be the fractional part of $x$, and set $\{x\}_{m}=m\{x / m\}$ for any $m \in \mathbb{Z}^{+}$.

Theorem 10. Let $m>1$ be an integer. Then for any $n \in \mathbb{Z}$ we have

$$
\left\lfloor\frac{n}{m}\right\rfloor+\left\lfloor\frac{6 n}{m}\right\rfloor \geqslant\left\lfloor\frac{2 n}{m}\right\rfloor+\left\lfloor\frac{2 n+1}{m}\right\rfloor+\left\lfloor\frac{3 n}{m}\right\rfloor .
$$

Proof. Let $A_{m}(n)$ denote the left-hand side of (6) minus the right-hand side. Then

$$
A_{m}(n)=\left\{\frac{2 n}{m}\right\}+\left\{\frac{2 n+1}{m}\right\}+\left\{\frac{3 n}{m}\right\}-\frac{1}{m}-\left\{\frac{n}{m}\right\}-\left\{\frac{6 n}{m}\right\},
$$

which only depends on $n$ modulo $m$. So, without any loss of generality we may simply assume that $n \in\{0, \ldots, m-1\}$. Hence $A_{m}(n) \geqslant 0$ if and only if

$$
\left\{\frac{2 n}{m}\right\}+\left\{\frac{2 n+1}{m}\right\}+\left\{\frac{3 n}{m}\right\} \geqslant \frac{n+1}{m} .
$$

(Note that $2 n+(2 n+1)+3 n-(n+1)=6 n$.) 
(6) is obvious when $n=0$. If $1 \leqslant n<m / 2$, then $\{2 n / m\}=2 n / m \geqslant(n+1) / m$ and hence ( 7 ) holds. In the case $n \geqslant m / 2,(7)$ can be simplified as

$$
\frac{3 n}{m}+\left\{\frac{3 n}{m}\right\} \geqslant 2
$$

which holds since $3 n \geqslant m+m / 2$.

By the above we have proved (6).

Theorem 11. Let $m \in \mathbb{Z}^{+}$and $k, n \in \mathbb{Z}$. Then we have

$$
\left\lfloor\frac{4 n+2 k+2}{m}\right\rfloor-\left\lfloor\frac{2 n+k+1}{m}\right\rfloor+2\left\lfloor\frac{k}{m}\right\rfloor-2\left\lfloor\frac{2 k}{m}\right\rfloor \geqslant\left\lfloor\frac{n}{m}\right\rfloor+\left\lfloor\frac{n-k+1}{m}\right\rfloor,
$$

unless $2 \mid m$ and $k \equiv n+1 \equiv m / 2(\bmod m)$ in which case the right-hand side of the inequality equals the left-hand side plus one.

Proof. Since

$$
(4 n+2 k+2)-(2 n+k+1)+2 k-2(2 k)=n+(n-k+1),
$$

(8) has the following equivalent form:

$$
\left\{\frac{4 n+2 k+2}{m}\right\}-\left\{\frac{2 n+k+1}{m}\right\}+2\left\{\frac{k}{m}\right\}-2\left\{\frac{2 k}{m}\right\} \leqslant\left\{\frac{n}{m}\right\}+\left\{\frac{n-k+1}{m}\right\} .
$$

Note that this only depends on $k$ and $n$ modulo $m$. So, without any loss of generality, we may simply assume that $k, n \in\{0, \ldots, m-1\}$.

Case $1 . k<m / 2$ and $\{2 n+k+1\}_{m}<m / 2$.

In this case, (9) can be simplified as

$$
\frac{n+2 k}{m}+\left\{\frac{n-k+1}{m}\right\} \geqslant\left\{\frac{2 n+k+1}{m}\right\}
$$

which is true since the left-hand side is nonnegative and $(n+2 k)+(n-k+1) \equiv 2 n+$ $k+1(\bmod m)$.

Case 2. $k<m / 2$ and $\{2 n+k+1\}_{m} \geqslant m / 2$.

In this case, (9) can be simplified as

$$
\frac{n+2 k}{m}+\left\{\frac{n-k+1}{m}\right\} \geqslant\left\{\frac{2 n+k+1}{m}\right\}-1
$$

which holds trivially since the right-hand side is negative.

Case $3 . k \geqslant m / 2$ and $\{2 n+k+1\}_{m}<m / 2$.

In this case, (9) can be simplified as

$$
\frac{n+2 k}{m}+\left\{\frac{n-k+1}{m}\right\} \geqslant 2+\left\{\frac{2 n+k+1}{m}\right\} .
$$


Since $(n+2 k)+(n-k+1)=2 n+k+1$, this is equivalent to

$$
n+2 k+\{n-k+1\}_{m} \geqslant 2 m .
$$

If $k>n+1$, then

$$
n+2 k+\{n-k+1\}_{m}=n+2 k+(n-k+1+m)=2 n+k+1+m \geqslant 2 m
$$

since $2 n+k+1>k \geqslant m / 2$ and $\{2 n+k+1\}_{m}<m / 2$.

Now assume that $k \leqslant n+1$. Clearly

$$
n+2 k+\{n-k+1\}_{m}=n+2 k+(n-k+1)=2 n+k+1 \geqslant 3 k-1 .
$$

If $k>m / 2$ then $3 k-1 \geqslant 3(m+1) / 2-1>3 m / 2$. If $k \leqslant n$ then $2 n+k+1>3 k \geqslant 3 m / 2$. So, except the case $k=n+1=m / 2$ we have

$$
n+2 k+\{n-k+1\}_{m}=2 n+k+1 \geqslant 3 m / 2
$$

and hence $n+2 k+\{n-k+1\}_{m}=2 n+k+1 \geqslant 2 m$ since $\{2 n+k+1\}_{m}<m / 2$.

When $k=n+1=m / 2$, the left-hand side of (9) minus the right-hand side equals

$$
\frac{m-2}{m}-\frac{m / 2-1}{m}+2 \frac{m / 2}{m}-\frac{m / 2-1}{m}=1 .
$$

Case $4 . k \geqslant m / 2$ and $\{2 n+k+1\}_{m} \geqslant m / 2$.

In this case, clearly $m \neq 1$, and (9) can be simplified as

$$
\frac{n+2 k}{m}+\left\{\frac{n-k+1}{m}\right\} \geqslant 1+\left\{\frac{2 n+k+1}{m}\right\}
$$

which is equivalent to

$$
n+2 k+\{n-k+1\}_{m} \geqslant m
$$

If $k \leqslant n+1$, then

$$
n+2 k+\{n-k+1\}_{m}=n+2 k+(n+1-k)=2 n+k+1 \geqslant 3 k-1 \geqslant \frac{3 m}{2}-1 \geqslant m .
$$

If $k>n+1$, then

$$
n+2 k+\{n-k+1\}_{m}=n+2 k+(n+1-k)+m=2 n+k+1+m>m .
$$

In view of the above, we have completed the proof of Theorem 11.

Theorem 12. Let $m \in \mathbb{Z}^{+}$and $k, n \in \mathbb{Z}$. Then we have

$$
\begin{aligned}
& \left\lfloor\frac{2 n+2 k}{m}\right\rfloor-\left\lfloor\frac{n+k}{m}\right\rfloor+2\left\lfloor\frac{k}{m}\right\rfloor-2\left\lfloor\frac{2 k}{m}\right\rfloor \\
& \geqslant 2\left\lfloor\frac{n}{m}\right\rfloor-\left\lfloor\frac{2 n+1}{m}\right\rfloor+\left\lfloor\frac{n-k+1}{m}\right\rfloor,
\end{aligned}
$$

unless $2 \mid m$ and $k \equiv n+1 \equiv m / 2(\bmod m)$ in which case the right-hand side of the inequality equals the left-hand side plus one. 
Proof. Since

$$
2 n+2 k-(n+k)+2 k-2(2 k)=2 n-(2 n+1)+(n-k+1),
$$

(10) is equivalent to the following inequality:

$$
\begin{aligned}
& \left\{\frac{2 n+2 k}{m}\right\}-\left\{\frac{n+k}{m}\right\}+2\left\{\frac{k}{m}\right\}-2\left\{\frac{2 k}{m}\right\} \\
& \leqslant 2\left\{\frac{n}{m}\right\}-\left\{\frac{2 n+1}{m}\right\}+\left\{\frac{n-k+1}{m}\right\} .
\end{aligned}
$$

As (11) only depends on $k$ and $n$ modulo $m$, without loss of generality we simply assume that $k, n \in\{0, \ldots, m-1\}$.

Case 1. $k<m / 2$ and $\{n+k\}_{m}<m / 2$.

In this case, (11) can be simplified as

$$
\frac{2 n+2 k}{m}+\left\{\frac{n-k+1}{m}\right\} \geqslant\left\{\frac{2 n+1}{m}\right\}+\left\{\frac{n+k}{m}\right\}
$$

which holds since

$$
\frac{2 n+2 k}{m}-\left\{\frac{n+k}{m}\right\}+\left\{\frac{n-k+1}{m}\right\} \geqslant 0
$$

and $2 n+2 k-(n+k)+(n-k+1)=2 n+1$.

Case 2. $k<m / 2$ and $\{n+k\}_{m} \geqslant m / 2$.

In this case, (11) can be simplified as

$$
\frac{2 n+2 k}{m}+\left\{\frac{n-k+1}{m}\right\} \geqslant\left\{\frac{2 n+1}{m}\right\}+\left\{\frac{n+k}{m}\right\}-1
$$

which holds since

$$
\frac{2 n+2 k}{m} \geqslant \frac{n+k}{m} \geqslant\left\{\frac{n+k}{m}\right\} \text { and }\left\{\frac{n-k+1}{m}\right\} \geqslant 0>\left\{\frac{2 n+1}{m}\right\}-1 .
$$

Case 3. $k \geqslant m / 2$ and $\{n+k\}_{m}<m / 2$.

In this case, we must have $n+k \geqslant m$ and hence $\{n+k\}_{m}=n+k-m$. Thus (11) can be simplified as

$$
\frac{n+k-m}{m}+\left\{\frac{n-k+1}{m}\right\} \geqslant\left\{\frac{2 n+1}{m}\right\}
$$

which holds trivially since $n+k-m+(n-k+1) \equiv 2 n+1(\bmod m)$.

Case $4 . k \geqslant m / 2$ and $\{n+k\}_{m} \geqslant m / 2$.

In this case, (11) can be simplified as

$$
\frac{2 n+2 k}{m}-\left\{\frac{n+k}{m}\right\}+\left\{\frac{n-k+1}{m}\right\} \geqslant 1+\left\{\frac{2 n+1}{m}\right\}
$$


which is equivalent to

$$
\frac{2(n+k)}{m}-\left\{\frac{n+k}{m}\right\}+\left\{\frac{n-k+1}{m}\right\} \geqslant 1
$$

since $2 n+2 k-(n+k)+(n-k+1)=2 n+1$.

Clearly (12) holds if $n+k \geqslant m$. If $n+k<m$ and $k>n+1$, then the left-hand side of the inequality (12) is

$$
\frac{n+k}{m}+\frac{n+1-k}{m}+1=\frac{2 n+1}{m}+1>1 .
$$

Now assume that $n+k<m$ and $k \leqslant n+1$. Then (12) is equivalent to $2 n+1 \geqslant m$. If $k \leqslant n$ then $2 n+1>2 k \geqslant m$. If $k=n+1 \neq m / 2$, then $k=n+1 \geqslant(m+1) / 2$ and hence $2 n+1=2(n+1)-1 \geqslant m$.

When $k=n+1=m / 2$, the left-hand side of (11) minus the right-hand side equals

$$
\frac{m-2}{m}-\frac{m-1}{m}+2 \frac{m / 2}{m}-2 \frac{m / 2-1}{m}+\frac{m-1}{m}=1 .
$$

Combining the discussion of the four cases we obtain the desired result.

\section{Proof of Theorem 1}

For a prime $p$, the $p$-adic evaluation of an integer $m$ is given by

$$
\nu_{p}(m)=\sup \left\{a \in \mathbb{N}: p^{a} \mid m\right\} .
$$

For a rational number $x=m / n$ with $m \in \mathbb{Z}$ and $n \in \mathbb{Z}^{+}$, we set $\nu_{p}(x)=\nu_{p}(m)-\nu_{p}(n)$ for any prime $p$. Note that a rational number $x$ is an integer if and only if $\nu_{p}(x) \geqslant 0$ for all primes $p$.

Proof of Theorem 1. (i) Fix $n \in \mathbb{Z}^{+}$, and define $A_{m}(n)$ for $m>1$ as in the proof of Theorem 10. Observe that

$$
Q:=\frac{\left(\begin{array}{c}
6 n \\
3 n
\end{array}\right)\left(\begin{array}{c}
3 n \\
n
\end{array}\right)}{(2 n+1)\left(\begin{array}{c}
2 n \\
n
\end{array}\right)}=\frac{n !(6 n) !}{(2 n) !(2 n+1) !(3 n) !} .
$$

So, for any prime $p$ we have

$$
\nu_{p}(Q)=\sum_{i=1}^{\infty} A_{p^{i}}(n) \geqslant 0
$$

by Theorem 10. Therefore $Q$ is an integer.

Choose $j \in \mathbb{Z}^{+}$such that $2^{j-1} \leqslant n<2^{j}$. As $2 n+1 \leqslant 2\left(2^{j}-1\right)+1<2^{j+1}$, we have

$$
\begin{aligned}
& \left\lfloor\frac{n}{2^{j+1}}\right\rfloor+\left\lfloor\frac{6 n}{2^{j+1}}\right\rfloor-\left\lfloor\frac{2 n}{2^{j+1}}\right\rfloor-\left\lfloor\frac{2 n+1}{2^{j+1}}\right\rfloor-\left\lfloor\frac{3 n}{2^{j+1}}\right\rfloor \\
= & \left\lfloor\frac{3 n}{2^{j}}\right\rfloor-\left\lfloor\frac{3 n}{2^{j+1}}\right\rfloor=\left\lfloor\frac{3 n+2^{j}}{2^{j+1}}\right\rfloor \geqslant\left\lfloor\frac{2 n+2^{j}}{2^{j+1}}\right\rfloor \geqslant 1 .
\end{aligned}
$$


Therefore

$$
\nu_{2}(Q)=\sum_{i=1}^{\infty} A_{2^{i}}(n) \geqslant A_{2^{j+1}}(n) \geqslant 1 .
$$

and hence $Q$ is even. This proves (1).

(ii) (2) and (3) are obvious in the case $k=0$. If $k>n+1$, then

$$
\left(\begin{array}{c}
2 n+k+1 \\
2 k
\end{array}\right)=\left(\begin{array}{c}
n+k+1 \\
2 k
\end{array}\right)=0
$$

and hence (2) and (3) hold trivially. Below we assume that $1 \leqslant k \leqslant n+1$.

Recall that for any nonnegative integer $m$ and prime $p$ we have

$$
\nu_{p}(m !)=\sum_{i=1}^{\infty}\left\lfloor\frac{m}{p^{i}}\right\rfloor .
$$

Since

$$
\frac{\left(\begin{array}{c}
4 n+2 k+2 \\
2 n+k+1
\end{array}\right)\left(\begin{array}{c}
2 n+k+1 \\
2 k
\end{array}\right)\left(\begin{array}{c}
2 n+k+1 \\
n
\end{array}\right)}{\left(\begin{array}{c}
2 k \\
k
\end{array}\right)}=\frac{(4 n+2 k+2) !(k !)^{2}}{(2 n+k+1) !((2 k) !)^{2} n !(n-k+1) !}
$$

and

$$
\frac{(2 n+1)\left(\begin{array}{c}
2 n \\
n
\end{array}\right) C_{n+k}\left(\begin{array}{c}
n+k+1 \\
2 k
\end{array}\right)}{\left(\begin{array}{c}
2 k \\
k
\end{array}\right)}=\frac{(2 n+1) !(2 n+2 k) !(k !)^{2}}{(n !)^{2}(n+k) !((2 k) !)^{2}(n-k+1) !},
$$

it suffices to show that for any prime $p$ we have

$$
\sum_{i=1}^{\infty} C_{p^{i}}(n, k) \geqslant 0 \text { and } \sum_{i=1}^{\infty} D_{p^{i}}(n, k) \geqslant 0,
$$

where

$$
\begin{aligned}
C_{m}(n, k)= & \left\lfloor\frac{4 n+2 k+2}{m}\right\rfloor-\left\lfloor\frac{2 n+k+1}{m}\right\rfloor+2\left\lfloor\frac{k}{m}\right\rfloor-2\left\lfloor\frac{2 k}{m}\right\rfloor \\
& -\left\lfloor\frac{n}{m}\right\rfloor-\left\lfloor\frac{n-k+1}{m}\right\rfloor
\end{aligned}
$$

and

$$
\begin{aligned}
D_{m}(n, k)= & \left\lfloor\frac{2 n+2 k}{m}\right\rfloor-\left\lfloor\frac{n+k}{m}\right\rfloor+2\left\lfloor\frac{k}{m}\right\rfloor-2\left\lfloor\frac{2 k}{m}\right\rfloor \\
& -2\left\lfloor\frac{n}{m}\right\rfloor+\left\lfloor\frac{2 n+1}{m}\right\rfloor-\left\lfloor\frac{n-k+1}{m}\right\rfloor .
\end{aligned}
$$

(a) By Theorem 11, $C_{p^{i}}(n, k) \geqslant 0$ unless $p=2$ and $k \equiv n+1 \equiv 2^{i-1}\left(\bmod 2^{i}\right)$ in which case $C_{2^{i}}(n, k)=-1$. Suppose that $k \equiv n+1 \equiv 2^{i-1}\left(\bmod 2^{i}\right), k=2^{i-1} k_{0}$ and $n+1=2^{i-1} n_{0}$, where $1 \leqslant k_{0} \leqslant n_{0}$ and $k_{0}$ and $n_{0}$ are odd. If $i \geqslant 2$, then

$$
C_{2^{i-1}}(n, k)=4 n_{0}+2 k_{0}-1-\left(2 n_{0}+k_{0}-1\right)+2 k_{0}-4 k_{0}-\left(n_{0}-1\right)-\left(n_{0}-k_{0}\right)=1
$$


and hence $C_{2^{i-1}}(n, k)+C_{2^{i}}(n, k)=1+(-1)=0$. So it remains to consider the case $k \equiv n+1 \equiv 1(\bmod 2)$.

Assume that $k$ is odd and $n$ is even. Write $k+1=2^{j} k_{1}$ and $n=2 n_{1}$ with $k_{1}, n_{1} \in \mathbb{Z}^{+}$ and $2 \nmid k_{1}$. Then it is easy to see that

$$
\begin{aligned}
C_{2^{j+1}}(n, k)= & \left\lfloor\frac{4 n_{1}}{2^{j}}\right\rfloor+k_{1}-\left\lfloor\frac{2 n_{1}-2^{j-1}+2^{j-1}\left(k_{1}-1\right)}{2^{j}}\right\rfloor \\
& +2\left\lfloor\frac{k_{1}}{2}\right\rfloor-2\left\lfloor\frac{2^{j} k_{1}-1}{2^{j}}\right\rfloor-\left\lfloor\frac{n_{1}}{2^{j}}\right\rfloor-\left\lfloor\frac{n_{1}+1-2^{j-1} k_{1}}{2^{j}}\right\rfloor \\
= & \left\lfloor\frac{4 n_{1}}{2^{j}}\right\rfloor+k_{1}-\left\lfloor\frac{2 n_{1}-2^{j-1}}{2^{j}}\right\rfloor-\frac{k_{1}+1}{2}+k_{1}-1-2\left(k_{1}-1\right) \\
& -\left\lfloor\frac{n_{1}}{2^{j}}\right\rfloor-\left\lfloor\frac{n_{1}+1+2^{j-1}}{2^{j}}\right\rfloor+\frac{k_{1}+1}{2} \\
= & 1+\left\lfloor\frac{n_{1}+\left(n_{1}+1+2^{j-1}\right)+\left(2 n_{1}-2^{j-1}\right)}{2^{j}}\right\rfloor \\
& -\left\lfloor\frac{n_{1}}{2^{j}}\right\rfloor-\left\lfloor\frac{n_{1}+1+2^{j-1}}{2^{j}}\right\rfloor-\left\lfloor\frac{2 n_{1}-2^{j-1}}{2^{j}}\right\rfloor \\
\geqslant & 1
\end{aligned}
$$

and hence $C_{2}(n, k)+C_{2^{j+1}}(n, k) \geqslant 0$.

By the above, we do have $\sum_{i=1}^{\infty} C_{p^{i}}(n, k) \geqslant 0$ for any prime $p$. So (2) holds.

(b) By Theorem 11, $D_{p^{i}}(n, k) \geqslant 0$ unless $p=2$ and $k \equiv n+1 \equiv 2^{i-1}\left(\bmod 2^{i}\right)$ in which case $D_{2^{i}}(n, k)=-1$. So, to prove (2) it suffices to find a positive integer $j$ such that $D_{2^{j}}(n, k) \geqslant 1$.

Clearly there is a unique positive integer $j$ such that $2^{j-1} \leqslant n+k<2^{j}$. Note that $k \leqslant(n+k) / 2<2^{j-1}$ and

$$
D_{2^{j}}(n, k)=1+\left\lfloor\frac{2 n+1}{2^{j}}\right\rfloor \geqslant 1 .
$$

This concludes the proof of (3).

The proof of Theorem 1 is now complete.

\section{Proof of Theorem 5}

Proof of Theorem 5. (i) We first prove (4). For $k, n \in \mathbb{N}$ define

$$
F(n, k)=\frac{(-1)^{n+k}(4 n+1)}{4^{3 n-k}}\left(\begin{array}{c}
2 n \\
n
\end{array}\right)^{2} \frac{\left(\begin{array}{c}
2 n+2 k \\
n+k
\end{array}\right)\left(\begin{array}{c}
n+k \\
2 k
\end{array}\right)}{\left(\begin{array}{c}
2 k \\
k
\end{array}\right)}
$$

and

$$
G(n, k)=\frac{(-1)^{n+k}(2 n-1)^{2}\left(\begin{array}{c}
2 n-2 \\
n-1
\end{array}\right)^{2}}{2(n-k) 4^{3(n-1)-k}}\left(\begin{array}{c}
2(n-1+k) \\
n-1+k
\end{array}\right) \frac{\left(\begin{array}{c}
n-1+k \\
2 k
\end{array}\right)}{\left(\begin{array}{c}
2 k \\
k
\end{array}\right)}
$$


Clearly $F(n, k)=G(n, k)=0$ if $n<k$. By [7],

$$
F(n, k-1)-F(n, k)=G(n+1, k)-G(n, k)
$$

for all $k \in \mathbb{Z}^{+}$and $n \in \mathbb{N}$.

Fix a positive integer $N$. Then

$$
\begin{aligned}
\sum_{n=0}^{N} F(n, 0)-F(N, N) & =\sum_{n=0}^{N} F(n, 0)-\sum_{n=0}^{N} F(n, N) \\
& =\sum_{k=1}^{N}\left(\sum_{n=0}^{N} F(n, k-1)-\sum_{n=0}^{N} F(n, k)\right) \\
& =\sum_{k=1}^{N} \sum_{n=0}^{N}(G(n+1, k)-G(n, k))=\sum_{k=1}^{N} G(N+1, k) .
\end{aligned}
$$

Note that

$$
\sum_{n=0}^{N} F(n, 0)=\sum_{n=0}^{N} \frac{4 n+1}{(-64)^{n}}\left(\begin{array}{c}
2 n \\
n
\end{array}\right)^{3}
$$

and

$$
F(N, N)=\frac{4 N+1}{4^{2 N}}\left(\begin{array}{c}
2 N \\
N
\end{array}\right)\left(\begin{array}{l}
4 N \\
2 N
\end{array}\right)=\frac{(4 N+1)(2 N+1)}{4^{2 N}}\left(\begin{array}{c}
2 N \\
N
\end{array}\right) C_{2 N} .
$$

Also,

$$
\begin{aligned}
\sum_{k=1}^{N} G(N+1, k) & =\frac{(2 N+1)^{2}}{2} \sum_{k=1}^{N} \frac{(-1)^{N+k+1}}{4^{3 N-k}}\left(\begin{array}{c}
2 N \\
N
\end{array}\right)^{2} C_{N+k} \frac{\left(\begin{array}{c}
N+k+1 \\
2 k
\end{array}\right)}{\left(\begin{array}{c}
2 k \\
k
\end{array}\right)} \\
& =\frac{2(2 N+1)\left(\begin{array}{c}
2 N \\
N
\end{array}\right)}{(-64)^{N}} \sum_{k=1}^{N}(-4)^{k-1} \frac{(2 N+1)\left(\begin{array}{c}
2 N \\
N
\end{array}\right) C_{N+k}\left(\begin{array}{c}
N+k+1 \\
2 k
\end{array}\right)}{\left(\begin{array}{c}
2 k \\
k
\end{array}\right)}
\end{aligned}
$$

and

$$
\begin{aligned}
\frac{\left(\begin{array}{c}
2 N \\
N
\end{array}\right) C_{N+1}\left(\begin{array}{c}
N+2 \\
2
\end{array}\right)}{\left(\begin{array}{c}
2 \\
1
\end{array}\right)}= & \left(\begin{array}{c}
2 N-1 \\
N-1
\end{array}\right)\left(\begin{array}{c}
2 N+2 \\
N+1
\end{array}\right) \frac{N+1}{2} \\
& =\left(\begin{array}{c}
2 N-1 \\
N-1
\end{array}\right)\left(\begin{array}{c}
2 N+1 \\
N+1
\end{array}\right)(N+1) \\
& =\left(\begin{array}{c}
2 N-1 \\
N-1
\end{array}\right)(2 N+1)\left(\begin{array}{c}
2 N \\
N
\end{array}\right) \\
& =2(2 N+1)\left(\begin{array}{c}
2 N-1 \\
N-1
\end{array}\right)^{2} \equiv 0(\bmod 2) .
\end{aligned}
$$

So, with the help of $(3)$ we see that $\sum_{n=0}^{N}(4 n+1)\left(\begin{array}{c}2 n \\ n\end{array}\right)^{3}(-64)^{N-n}$ is divisible by $4(2 N+$ 1) $\left(\begin{array}{c}2 N \\ N\end{array}\right)$. 
(ii) Now we turn to the proof of (5).

For $n, k \in \mathbb{N}$, define

$$
F(n, k):=\frac{(-1)^{n+k}(20 n-2 k+3)}{4^{5 n-k}} \cdot \frac{\left(\begin{array}{c}
2 n \\
n
\end{array}\right)\left(\begin{array}{c}
4 n+2 k \\
2 n+k
\end{array}\right)\left(\begin{array}{c}
2 n+k \\
2 k
\end{array}\right)\left(\begin{array}{c}
2 n-k \\
n
\end{array}\right)}{\left(\begin{array}{c}
2 k \\
k
\end{array}\right)} .
$$

and

$$
G(n, k):=\frac{(-1)^{n+k}}{4^{5 n-4-k}} \cdot \frac{n\left(\begin{array}{c}
2 n \\
n
\end{array}\right)\left(\begin{array}{c}
4 n+2 k-2 \\
2 n+k-1
\end{array}\right)\left(\begin{array}{c}
2 n+k-1 \\
2 k
\end{array}\right)\left(\begin{array}{c}
2 n-k-1 \\
n-1
\end{array}\right)}{\left(\begin{array}{c}
2 k \\
k
\end{array}\right)} .
$$

Clearly $F(n, k)=G(n, k)=0$ if $n<k$. By [22],

$$
F(n, k-1)-F(n, k)=G(n+1, k)-G(n, k)
$$

for all $k \in \mathbb{Z}^{+}$and $n \in \mathbb{N}$.

Fix a positive integer $N$. As in part (i) we have

$$
\sum_{n=0}^{N} F(n, 0)-F(N, N)=\sum_{k=1}^{N} G(N+1, k) .
$$

Observe that

$$
\sum_{n=0}^{N} F(n, 0)=\sum_{n=0}^{N} \frac{20 n+3}{\left(-2^{10}\right)^{n}}\left(\begin{array}{c}
2 n \\
n
\end{array}\right)^{2}\left(\begin{array}{c}
4 n \\
2 n
\end{array}\right)
$$

and

$$
F(N, N)=\frac{18 N+3}{2^{8 N}}\left(\begin{array}{c}
6 N \\
3 N
\end{array}\right)\left(\begin{array}{c}
3 N \\
N
\end{array}\right)
$$

Also,

$$
\sum_{k=1}^{N} G(N+1, k)=\frac{2(2 N+1)\left(\begin{array}{c}
2 N \\
N
\end{array}\right)}{\left(-2^{10}\right)^{N}} \sum_{k=1}^{N}(-4)^{k-1} \frac{\left(\begin{array}{c}
4 N+2 k+2 \\
2 N+k+1
\end{array}\right)\left(\begin{array}{c}
2 N+k+1 \\
2 k
\end{array}\right)\left(\begin{array}{c}
2 N-k+1 \\
N
\end{array}\right)}{\left(\begin{array}{c}
2 k \\
k
\end{array}\right)}
$$

Note that

$$
\frac{\left(\begin{array}{c}
4 N+4 \\
2 n+2
\end{array}\right)\left(\begin{array}{c}
2 N+2 \\
2
\end{array}\right)\left(\begin{array}{c}
2 N \\
N
\end{array}\right)}{\left(\begin{array}{l}
2 \\
1
\end{array}\right)}=2\left(\begin{array}{c}
4 N+3 \\
2 N+1
\end{array}\right)\left(\begin{array}{c}
2 N+2 \\
2
\end{array}\right)\left(\begin{array}{c}
2 N-1 \\
N-1
\end{array}\right) \equiv 0(\bmod 2) .
$$

Applying (2) we see that $\left(-2^{10}\right)^{N} \sum_{k=1}^{N} G(N+1, k)$ is a multiple of $4(2 N+1)\left(\begin{array}{c}2 N \\ N\end{array}\right)$. By (1),

$$
\left(-2^{10}\right)^{N} \frac{18 N+3}{2^{8 N}}\left(\begin{array}{c}
6 N \\
3 N
\end{array}\right)\left(\begin{array}{c}
3 N \\
N
\end{array}\right)
$$

is divisible by $8(2 N+1)\left(\begin{array}{c}2 N \\ N\end{array}\right)$. Therefore

$$
\sum_{n=0}^{N}(20 n+3)\left(\begin{array}{c}
2 n \\
n
\end{array}\right)^{2}\left(\begin{array}{c}
4 n \\
2 n
\end{array}\right)\left(-2^{10}\right)^{N-n}
$$

is a multiple of $4(2 N+1)\left(\begin{array}{c}2 N \\ N\end{array}\right)$.

Combining the above, we have completed the proof of Theorem 5. 


\section{References}

[1] T. Amdeberhan and D. Zeilberger. Hypergeometric series acceleration via the WZ method. Electron. J. Combin., 4(2):\#R3, 1997.

[2] N. D. Baruah and B. C. Berndt. Eisenstein series and Ramanujan-type series for $1 / \pi$. Ramanujan J., 23:17-44, 2010.

[3] N. D. Baruah, B. C. Berndt and H. H. Chan. Ramanujan's series for $1 / \pi$ : a survey. Amer. Math. Monthly, 116:567-587, 2009.

[4] B. C. Berndt. Ramanujan's Notebooks, Part IV. Springer, New York, 1994.

[5] N. J. Calkin. Factors of sums of powers of binomial coefficients. Acta Arith., 86:17-26, 1998.

[6] H. Q. Cao and H. Pan. Factors of alternating binomial sums. Adv. in Appl. Math., 45:96$107,2010$.

[7] S. B. Ekhad and D. Zeilberger. A WZ proof of Ramanujan's formula for $\pi$. In Geometry, Analysis, and Mechanics (J. M. Rassias, ed.), pages 107-108. World Sci., Singapore, 1994.

[8] J. Guillera. Some binomial series obtained by the WZ method. Adv. in Appl. Math., 29:599-603, 2002.

[9] V. J. W. Guo, F. Jouhet and J. Zeng. Factors of alternating sums of products of binomial and $q$-binomial coefficients. Acta Arith., 127:17-31, 2007.

[10] E. Mortenson. A p-adic supercongruence conjecture of van Hamme. Proc. Amer. Math. Soc., 136:4321-4328, 2008.

[11] M. Petkovšek, H. S. Wilf and D. Zeilberger. $A=B$. A K Peters, Wellesley, 1996.

[12] S. Ramanujan. Modular equations and approximations to $\pi$. Quart. J. Math. (Oxford) (2), 45:350-372, 1914.

[13] R. P. Stanley. Enumerative Combinatorics, Vol. 2. Cambridge Univ. Press, Cambridge, 1999.

[14] Z.-W. Sun. Open conjectures on congruences. http://arxiv.org/abs/0911.5665.

[15] Z.-W. Sun. Binomial coefficients, Catalan numbers and Lucas quotients. Sci. China Math., 53:2473-2488, 2010.

[16] Z.-W. Sun. Sequences A176285, A176477, A176898, in N.J.A. Sloane's OEIS. http://oeis.org/.

[17] Z.-W. Sun. A message to Number Theory List. April 4, 2010. Available from http://listserv.nodak. edu/cgi-bin/wa. exe?A2=NMBRTHRY;616ebfe6 . 1004

[18] Z.-W. Sun and R. Tauraso. New congruences for central binomial coefficients. Adv. in Appl. Math., 45:125-148, 2010.

[19] Z.-W. Sun and R. Tauraso. On some new congruences for binomial coefficients. Int. J. Number Theory, 7(3):645-662, 2011.

[20] L. van Hamme. Some conjectures concerning partial sums of generalized hypergeometric series. In p-adic Functional Analysis (Nijmegen, 1996), volume 192 of Lecture Notes in Pure and Appl. Math., pages 223-236. Dekker, 1997.

[21] D. Zeilberger. Closed form (pun intended!). Contemporary Math., 143:579-607, 1993.

[22] W. Zudilin. Ramanujan-type supercongruences. J. Number Theory, 129:1848-1857, 2009.

THE ELECTRONic JOURNAL OF COMBINATORICS 20(1) (2013), \#P9 